\title{
Probing the Fusion-Active Structures of Envelope of Human T-cell Leukaemia Virus Type-I with Conformation-Specific Monoclonal Antibodies
}

\author{
Antonis Mirsaliotis, Kulpash Nurkiyanova, C Sharon Kuo and \\ David W Brighty*‡
}

Address: The Biomedical Research Centre, The University of Dundee, DD1 9SY, Scotland

Email: David W Brighty* - david.brighty@cancer.org.uk

* Corresponding author $¥$ Presenting author

from 2005 International Meeting of The Institute of Human Virology Baltimore, USA, 29 August - 2 September 2005

Published: 8 December 2005

Retrovirology 2005, 2(SuppI I):PI9 doi:I0.I I86/1742-4690-2-SI-PI9

Infection of cells by human T cell leukaemia virus (HTLV1 ) is mediated by the viral envelope glycoproteins. The gp46 surface glycoprotein binds to the cell surface receptor Glut-1, allowing the transmembrane glycoprotein to initiate fusion of the viral and cellular membranes. In the absence of membrane fusion viral entry into the host cell cannot occur. Thus, envelope is a prime target for the development of anti-viral vaccines and small-molecule antagonists of viral infection. Indeed, we have shown that HTLV-1 infection can be blocked at all stages of the entry process including, viral attachment, primary receptor binding and the post-binding steps of viral entry. To extend our studies, we have expressed recombinant protein fragments that mimic the core-coiled-coil region and six-helix bundle of fusion-active HTLV-1 envelope. Using these recombinant proteins as immunogens we have generated monoclonal antibodies (mAbs) against the fusionactive and post-fusion conformations of HTLV-1 envelope. Most importantly, we have now used these conformation-specific mAbs to probe the events that culminate in membrane fusion. We demonstrate that these monoclonal antibodies can be used to detect viral envelope on infected cells and to monitor the process of cell-to-cell viral transfer. Our recent results will be presented, and the implications of our results for HTLV-1 pathogenesis will be discussed. 